\title{
Improved Thermoelectric Performances of Oxide-Containing $\mathrm{FeSi}_{2}$
}

\author{
Sunao Sugihara ${ }^{1}$ and Kentaro Morikawa ${ }^{2}$ \\ ${ }^{1}$ Department of Materials Science and Technology, Shonan Institute of Technology, Fujisawa 251-8511, Japan \\ ${ }^{2}$ Japan Finechem Co. Inc., Hiratsuka 254-0016, Japan
}

Because of its chemical stability and low cost, iron silicide is a promising thermoelectric material for use at high temperatures. Its performance, however, is poor compared with that of BiTe, PbTe, or SiGe, which are popular thermoelectric materials. We produced n-type $\mathrm{FeSi}_{2}$ samples containing various oxides, which showed a good thermoelectric performance. We attempted to unify the parameters attributing to thermoelectric performance, and the electrical resistivity decreased while an adequate Seebeck coefficient was retained and the thermal conductivity was reduced. This results in a greater value of the Seebeck coefficient, particularly on addition of $\mathrm{Sm}_{2} \mathrm{O}_{3}$; the resulting figure of merit ZT was 0.56 at $868 \mathrm{~K}$. Addition of $\mathrm{Er}_{2} \mathrm{O}_{3}$ gave a $\mathrm{ZT}$ value of 0.54 at $877 \mathrm{~K}$, and the material showed a lower thermal conductivity of $2-$ $2.5 \mathrm{~W} / \mathrm{m} \cdot \mathrm{K}$. [doi:10.2320/matertrans.E-M2011812]

(Received November 24, 2010; Accepted April 22, 2011; Published June 15, 2011)

Keywords: Seebeck coefficient, electrical resistivity, thermal conductivity, standard reduction potential, Onsager relation

\section{Introduction}

It is vital that systems be developed for the production of energy in an environmentally friendly manner. Many studies have been carried out on thermoelectric power, by which thermal energy in the form of a difference in temperature is converted directly into electrical energy. $\mathrm{Bi}_{2} \mathrm{Te}_{3}, \mathrm{PbTe}$, and $\mathrm{SiGe}$ are currently popular as thermoelectric materials; however, these materials suffer drawbacks in that they are manufactured from expensive and toxic raw materials. It is therefore important to develop alternative thermoelectric materials that are inexpensive and environmentally friendly.

The performance of thermoelectric materials is evaluated by using the expression $\mathrm{Z}=\alpha^{2} / \rho \kappa$, where $\mathrm{Z}$ is the figure of merit, $\alpha$ is the Seebeck coefficient, $\rho$ is the electrical resistivity, and $\kappa$ is the thermal conductivity. Another method of evaluation involves $\mathrm{ZT}$, where $\mathrm{T}$ is the temperature, as there is an optimal temperature for a thermoelectric material at which it shows its best performance. $\beta-\mathrm{FeSi}_{2}$ is suitable for use at high-temperatures $(1130 \mathrm{~K}),{ }^{1)}$ is an environmentally friendly resource, and can be fabricated in a U-shaped mould in $\mathrm{p}$ - and n-types by doping with $\mathrm{Mn}$ and Co, respectively. ${ }^{2)}$ However, $\beta-\mathrm{FeSi}_{2}$ has a comparatively high thermal conductivity. In previous studies, oxides of trivalent metals, such as $\mathrm{La}_{2} \mathrm{O}_{3}$ and $\mathrm{Y}_{2} \mathrm{O}_{3}$, and tetravalent metals, such as $\mathrm{TiO}_{2}$ and $\mathrm{LaSrMnO}_{3}$ (LSMO) have been added to n-type $\mathrm{FeSi}_{2}$ to improve its performance. ${ }^{3-5)}$ Ito et al. developed a process that uses mechanical alloying, ${ }^{6}$ ) but they did not succeed in achieving any improvement, reporting a figure of merit of only $0.1 \times 10^{-3}$. Our goal was to improve thermoelectric properties of n-type doped samples of $\mathrm{FeSi}_{2}$, such as $\left(\mathrm{Fe}_{0.95} \mathrm{Co}_{0.05}\right) \mathrm{Si}_{2}$. By means of calculations from first principles, we previously studied the development of such materials, taking into consideration their electronic structures. ${ }^{7)}$ We identified the existence of an impurity energy level in the gap between the conduction band and the valence band. Furthermore, addition of the pentavalent metal oxide $\mathrm{Ta}_{2} \mathrm{O}_{5}$ resulted in an increase in the figure of merit for $\mathrm{FeSi}_{2}$ through a decrease in its electrical resistivity and thermal conductivity. ${ }^{8)}$
Here, we report the achievement of a marked improvement in the figure of merit for n-type $\mathrm{FeSi}_{2}$ through the addition of the rare earth oxides such as $\mathrm{Sm}_{2} \mathrm{O}_{3}$ and $\mathrm{Er}_{2} \mathrm{O}_{3}$; the electrical resistivity decreased while an adequate Seebeck coefficient was retained and the thermal conductivity was reduced.

\section{Experimental Details}

Powdered metallic $\mathrm{Fe}, \mathrm{Si}$, and Co were mixed to form $\mathrm{Fe}_{0.95} \mathrm{Co}_{0.05} \mathrm{Si}_{2}$, an n-type semiconductor, and the material was ball-milled for $24 \mathrm{~h}$ then melted in vacuum for $2 \mathrm{~h}$ at $1523 \mathrm{~K}$; the resulting molten ingot was crushed to an average size of $20 \mu \mathrm{m}$ and mixed with an oxide power $\left(\mathrm{Sm}_{2} \mathrm{O}_{3}\right.$, $\mathrm{Er}_{2} \mathrm{O}_{3}$, or $\mathrm{Ta}_{2} \mathrm{O}_{5}$ ) by using $3 \mathrm{~mm}$ YTZ balls in a ball mill for $24 \mathrm{~h}$ to give the required oxide-containing iron silicide specimen. The mixed powder was then pressed at $245 \mathrm{MPa}$, sintered at $1413 \mathrm{~K}$, and finally annealed at $1033 \mathrm{~K}$ to give doped samples of $\beta-\mathrm{Fe}_{0.95} \mathrm{Co}_{0.05} \mathrm{Si}_{2}$. The thermoelectric power (Seebeck coefficient) and the electrical resistivity were measured by the four-probe method. The thermal conductivity was measured by the laser-flash method. Microstructure analysis was performed by X-ray diffraction (XRD), scanning electron microscopy, and electron-probe microanalysis to identify the regions in which the oxides existed in a crystalline form.

\section{Results and Discussion}

\subsection{Thermoelectric power and electrical resistivity}

Figures 1 and 2 show the temperature dependences of the thermoelectric power (Seebeck coefficient) for samples of $\mathrm{Fe}_{0.95} \mathrm{Co}_{0.05} \mathrm{Si}_{2}$ containing 0,1 or 10 mass\% of $\mathrm{Sm}_{2} \mathrm{O}_{3}$ and $\mathrm{Er}_{2} \mathrm{O}_{3}$, respectively. The Seebeck coefficient decreased with increasing oxide content by -230 to $-210 \mu \mathrm{V} / \mathrm{K}$ at 873 to $673 \mathrm{~K}$, respectively. The presence of the $\beta$-phase of $\mathrm{FeSi}_{2}$ is necessary for the material to display a thermoelectric effect; however, other phases, such as the $\varepsilon$-phase and $\mathrm{SmFe}_{1.4}$, are formed when the oxide content is increased, which may result in the observed decrease in the Seebeck coefficient. $\mathrm{In}_{2} \mathrm{O}_{3}$ containing sample, XRD analysis showed the presence of the $\varepsilon$-phase with no other phases. 


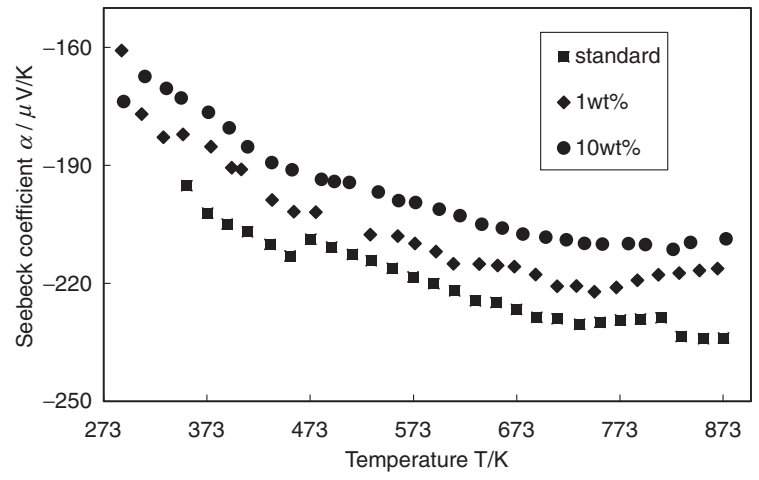

Fig. 1 Temperature dependence of the Seebeck coefficient of $\mathrm{FeSi}_{2}$ with $\mathrm{Sm}_{2} \mathrm{O}_{3}$.

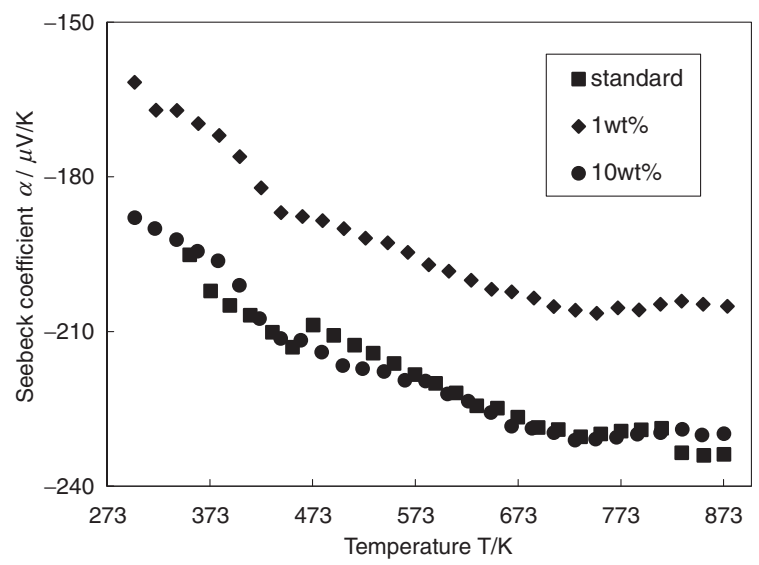

Fig. 2 Temperature dependence of the Seebeck coefficient of $\mathrm{FeSi}_{2}$ with $\mathrm{Er}_{2} \mathrm{O}_{3}$.

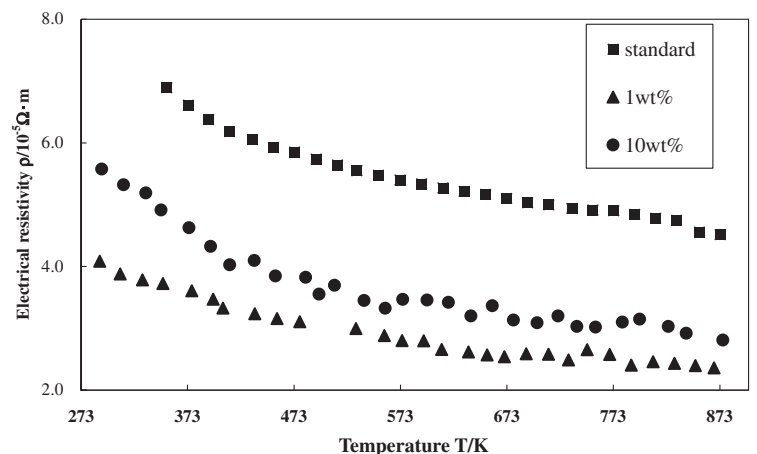

Fig. 3 Temperature dependence of the electrical resistivity of $\mathrm{FeSi}_{2}$ with $\mathrm{Sm}_{2} \mathrm{O}_{3}$.

The electrical resistivities of $\mathrm{Sm}_{2} \mathrm{O}_{3}$ - and $\mathrm{Er}_{2} \mathrm{O}_{3}$-containing samples are shown in Figs. 3 and 4, respectively. Addition of the oxide helped to reduce the electrical resistivity by about $50 \%$ for both samples compared with a standard sample with no added oxide. The cause of this reduction in resistivity is believed to be the local deformation of the crystal caused by polarization of lattice; as a result of this lattice-induced polarization, electrons mobility is increased in the defects in the oxide doped-material. Another effect of adding oxide was a slight tendency for the electrical resistivity to decrease with increasing temperature. As shown

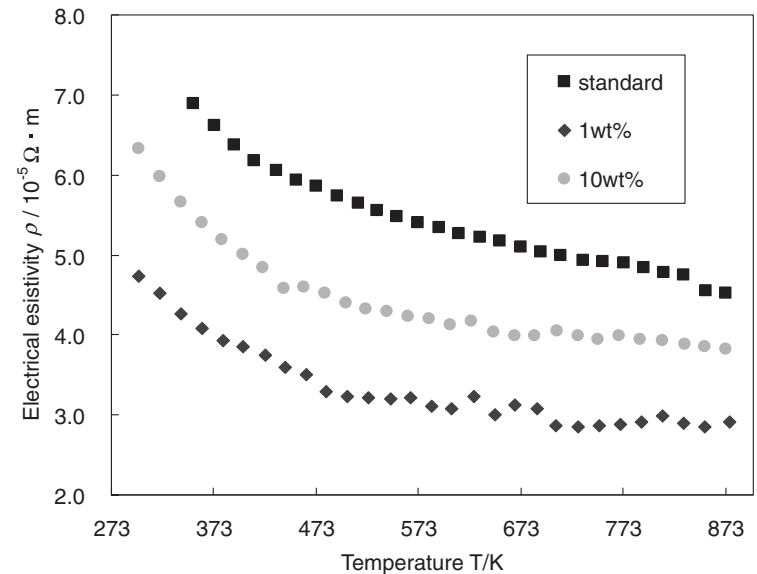

Fig. 4 Temperature dependence of the electrical resistivity of $\mathrm{FeSi}_{2}$ with $\mathrm{Er}_{2} \mathrm{O}_{3}$.

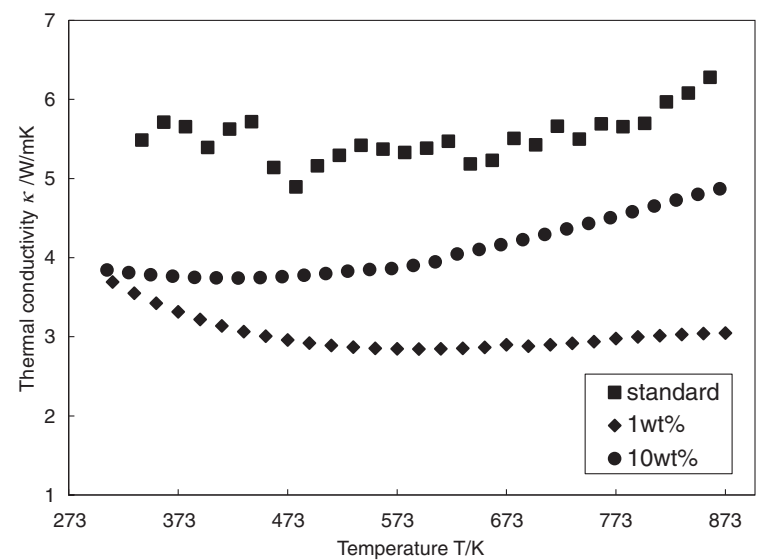

Fig. 5 Temperature dependence of the thermal conductivity of $\mathrm{FeSi}_{2}$ with $\mathrm{Sm}_{2} \mathrm{O}_{3}$.

in Figs. 1 and 2, the absolute values of the Seebeck coefficient decreased with increasing temperature. An increase in the absolute Seebeck coefficient and a decrease in electrical resistivity with increasing temperature are favorable for thermoelectric materials, as discussed above.

\subsection{Thermal conductivity}

The third important property of a thermoelectric material is its thermal conductivity. The temperature dependences of the thermal conductivity of the $\mathrm{Sm}_{2} \mathrm{O}_{3}$ - and $\mathrm{Er}_{2} \mathrm{O}_{3}$ containing samples are shown in Figs. 5 and 6, respectively. The value of the thermal conductivity can be calculated from the following equation, which is based on the WiedemannFranz law:

$$
L=\frac{\kappa_{\mathrm{e}}}{\sigma T}=\frac{\pi^{2}}{3}\left(\frac{K_{\mathrm{B}}}{e}\right)^{2}=2.45 \times 10^{-8} \mathrm{~W} \cdot \Omega / \mathrm{K}^{2}
$$

where $L$ is the Lorentz number, $\sigma$ is the electrical conductivity, $\kappa_{\mathrm{e}}$ is the thermal conductivity due to electrons, $K_{\mathrm{B}}$ is the Boltzmann constant, and $e$ is the charge on the electron.

The thermal conductivity is described by eq. (1) because the measured thermal conductivity $\kappa$ is the sum of two parts $\kappa_{\mathrm{e}}+\kappa_{\mathrm{ph}}$, where $\kappa_{\mathrm{ph}}$ is the thermal conductivity due to lattice vibrations (i.e., phonons). If we examine the contribution of 


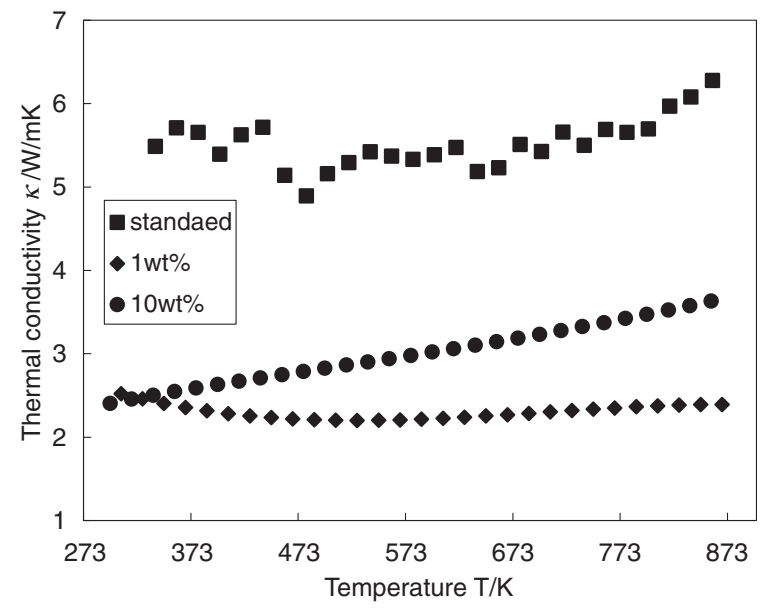

Fig. 6 Temperature dependence of the thermal conductivity of $\mathrm{FeSi}_{2}$ with $\mathrm{Er}_{2} \mathrm{O}_{3}$.

electrons to the electrical resistivity and Seebeck coefficient relating to thermal conductivity in the sample of containg$\mathrm{Sm}_{2} \mathrm{O}_{3}$, for example; the ratio of $\kappa_{\mathrm{ph}} / \kappa_{\mathrm{e}}$ for $\left(\mathrm{Fe}_{0.95} \mathrm{Co}_{0.05}\right) \mathrm{Si}_{2}$ is larger (13) than is the case for oxide-containing samples (2.9-6.7) leading to decreases of Seebeck coefficient and electrical resistivity. This suggests that $\kappa_{\mathrm{e}}$ should be larger, although lower thermal conductivity $\kappa$ is better, as shown in paragraph 3.4.

\subsection{Standard reduction potential}

The standard reduction potential (SRP) appears to be an essential property of thermoelectric materials. The SRP for constituent atoms in a crystal can be summarized by considering the potential required to convert the atoms between their various oxidation states. In the case of $\mathrm{Fe}$, for instances, the values are as follows: ${ }^{9)}$

$$
\mathrm{Fe}^{3+} \rightarrow \mathrm{Fe}^{2+}(+0.771 \text { volts }) \quad \mathrm{Fe}^{2+} \rightarrow \mathrm{Fe} \text { (-0.44 volts). }
$$

The corresponding values for Co are:

$$
\mathrm{Co}^{3+} \rightarrow \mathrm{Co}^{2+}(+1.92 \text { volts }) \quad \mathrm{Co}^{2+} \rightarrow \mathrm{Co}(-0.277 \text { volts }) \text {. }
$$

For $\mathrm{Fe}^{3+}$ and $\mathrm{Co}^{2+}$, the average SRP is calculated from the following equation:

$$
\zeta_{i j}=(0.771-0.277) / 2=|0.247| \cong 0.25 \text { volts. }
$$

On the basis of our unifying model, $\alpha / \rho$ and $\alpha / \kappa$ are plotted against the reduction potential, and the results are shown in Figs. 7 and 8, respectively. The increase in the average value of the integrated reduction potential $\zeta_{i j}$ between two atoms in the system suggests an increase in the logarithmic ratio of the thermoelectric power and the electrical resistivity, $\log (\alpha / \rho)$, as shown in Fig. 7. Furthermore, the combination of the thermal conductivity, $\log (\alpha / \kappa)$ suggests that a maximum value of the ratio may exist as a function of the reduction potential $\left(\zeta_{i j}\right)$, as described in Fig. 8. In these figures, we plotted other researchers' data for $\mathrm{Cu}$-doped $\mathrm{FeSi}_{2},{ }^{10)}$ the Ti-based half-Heusler structure ${ }^{11)}$ and ZrNiSn half-Heusler. ${ }^{12)}$ Taken together, these results suggest that $\alpha, \rho$ and $\kappa$ in combination play important roles in relation to the design of state-of-the-art TE material if the value of $\zeta_{i j}$ is introduced.

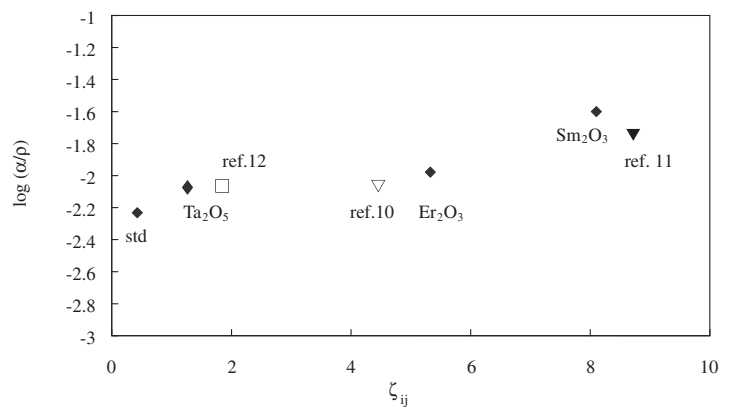

Fig. 7 Dependence of $\log (\alpha / \rho)$ on the reduction potential of oxidecontaining $\mathrm{FeSi}_{2}$ systems (Ref. 10): $\mathrm{Cu}$-doped $\mathrm{FeSi}_{2}$; Ref. 11): Ti-based half-Heusler structure; Ref. 12): $\mathrm{ZrNiSn}$ half-Heusler). $\zeta_{i j}$ is defined in the difference of reduction potential between each two atoms.

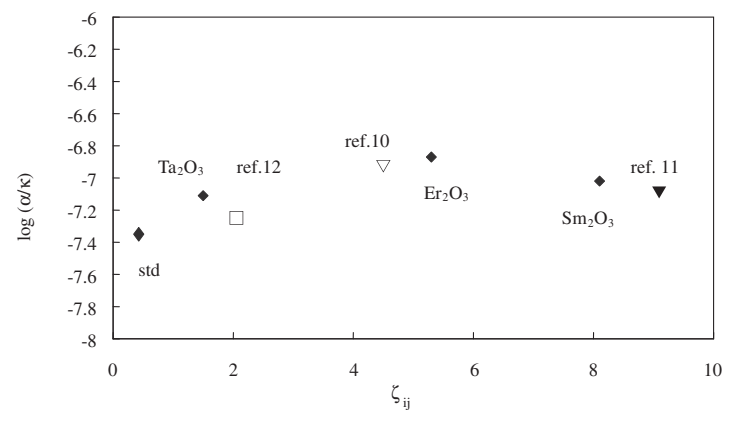

Fig. 8 Dependence of $\log (\alpha / \kappa)$ on the reduction potential of oxidecontaining $\mathrm{FeSi}_{2}$ systems. (Ref. 10): Cu-doped $\mathrm{FeSi}_{2}$; Ref. 11): Ti-based half-Heusler structure, Ref. 12): $\mathrm{ZrNiSn}$ half-Heusler). $\zeta_{i j}$ is defined in the difference of reduction potential between each two atoms.

\subsection{Figure of merit (thermoelectric performance)}

The thermoelectric performance is generally evaluated by means of the following equation:

$$
Z=\frac{\alpha^{2}}{\rho \kappa}
$$

where $Z$ is the figure of merit, $\alpha$ is the Seebeck coefficient $\left(\mathrm{VK}^{-1}\right), \rho$ is the electrical resistivity $(\Omega \mathrm{m})$, and $\kappa$ is the thermal conductivity $\left(\mathrm{Wm}^{-1} \mathrm{~K}^{-1}\right)$. In addition to evaluation of the figure of merit by means of eq. (2), the value of ZT is can also be used as a measure of the performance of a thermoelectric material at the optimum temperature where the material shows its best performance. Mahan et al. ${ }^{13)}$ reviewed the figure of merit for iron silicide in thermoelectric materials; according to their report, the value of $\mathrm{ZT}$ for $\mathrm{FeSi}_{2}$ is $0.2-0.3$. The thermoelectric properties of materials can be controlled by altering three properties simultaneously. ${ }^{14)}$ The values of $\kappa_{\mathrm{e}}$ and the Seebeck coefficient of iron silicide are markedly dependent on the temperature, whereas the electrical resistivity is relatively independent of the temperature.

It is not actually possible to express the Seebeck coefficient in terms of a simple factor because, in addition to the energy gap, the interaction of electrons with phonons (polarons) may also play a role in thermoelectric power. In an ionic lattice such as an oxide, electrons are mainly scattered by electromagnetic polar vibrations, which cause positive and negative ions to move in opposite directions in 


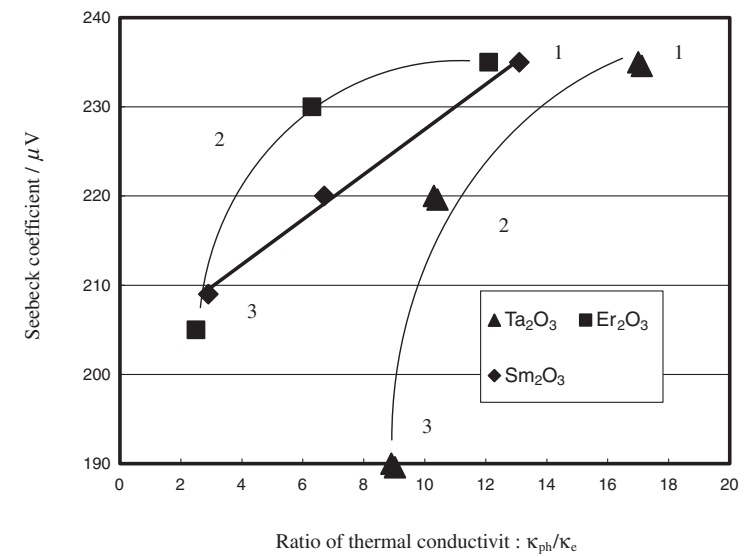

Fig. 9 Dependence of Seebeck coefficient against $\kappa_{\mathrm{ph}} / \kappa_{\mathrm{e}}$ for each oxidecontaining $\mathrm{FeSi}_{2}$. No. 1; non oxide-containing sample (standard), No. 2; 1 mass $\%$, No. $3 ; 10$ mass $\%$.

the lattice. This leads to the appearance of very powerful electric fields that deflect the moving electrons. Therefore, the change in the free path length of electrons $\left(l_{\text {pol }}\right)$ with a kinetic energy $(\varepsilon)$ is given by the following equation, derived by Pekar in Ref. 15), $l_{\text {pol }} \propto \varepsilon^{2}$. Pekar's theory is found to be well substantiated in the case of ionic semiconductors when the electrons diffuse simultaneously with the polarization of the surrounding medium.

An example of the Onsager relation ${ }^{16)}$ can be formulated for the transport coefficient for thermoelectric phenomena. ${ }^{17)}$ It is important that the relationship between heat flow and electric current should be considered when the gradients of the electrochemical potential and temperature can be driving forces. The flow of lattice vibrations (phonons) is related to the internal energy (heat), $\mathrm{J}_{\text {th }}$, and the force, $\mathrm{X}_{\mathrm{th}}$, with which the temperature gradient affects the phonons. Furthermore, the flow of electrons is related to the kinetic energy (electric current), $\mathrm{J}_{\mathrm{m}}$, and force, $\mathrm{X}_{\mathrm{m}}$ with which the temperature gradient affects the electrons.

The following equations are formulated with the transport coefficient $\left(\mathrm{L}_{i j}\right)$.

$$
\begin{aligned}
& \mathrm{J}_{\mathrm{m}}=\mathrm{L}_{11} \mathrm{X}_{\mathrm{m}}+\mathrm{L}_{12} \mathrm{X}_{\mathrm{th}} \\
& \mathrm{J}_{\mathrm{th}}=\mathrm{L}_{21} \mathrm{X}_{\mathrm{m}}+\mathrm{L}_{22} \mathrm{X}_{\mathrm{th}}
\end{aligned}
$$

When the thermal conductivity is reduced, the force component $\mathrm{L}_{21} \mathrm{X}_{\mathrm{m}}$ can be small in the second equation (corresponding to $\kappa_{\mathrm{ph}}$ ), leading to a reduction in thermoelectric power, as shown in Fig. 9. Therefore, a reduction in the thermal conductivity is not necessarily beneficial. As another factor, it is important to maintain a high kinetic energy of electrons, which, in turn, affects $\mathrm{L}_{12}$ (which may be related to the Seebeck coefficient) and $L_{21}$ of the electron energy associated with phonons (which is related to the electrical resistivity and the Seebeck coefficient, as mentioned above). This results in a lower value of the $\rho$ as a result of enhancement of $\kappa_{\mathrm{e}}$, as shown in Fig. 10. This suggested, therefore, that the value of $\kappa_{\mathrm{ph}}$ should be reduced or that of $\kappa_{\mathrm{e}}$ should be increased. Hasaka et al. ${ }^{10)}$ reported the effects of the addition of a metal $(\mathrm{Ag}$ or $\mathrm{Cu})$ to $\beta-\mathrm{FeSi}_{2}$ and discussed the figure of merit at $300 \mathrm{~K}$. The compound $\left(\mathrm{Fe}_{0.97} \mathrm{Co}_{0.03} \mathrm{Si}_{x}\right)_{100-y-z} \mathrm{Ag}_{y} \mathrm{Cu}_{z}$ did not show any marked

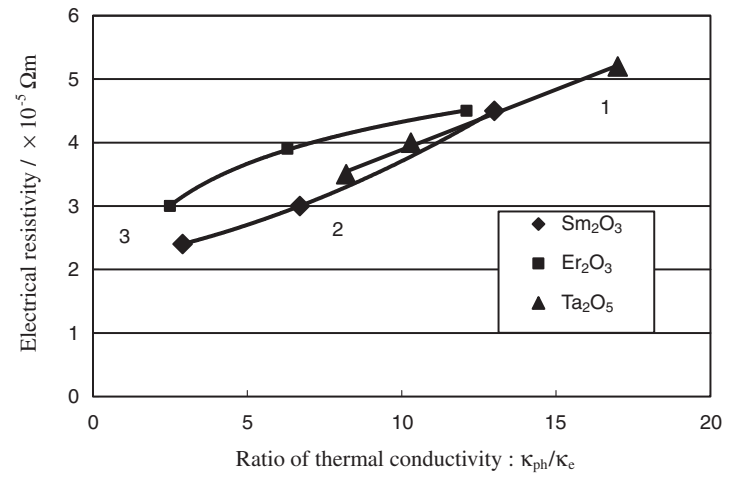

Fig. 10 Dependence of electrical resistivity against $\kappa_{\mathrm{ph}} / \kappa_{\mathrm{e}}$ for each oxidecontaining $\mathrm{FeSi}_{2}$. The numbers near lines indicate same meaning as in Fig. 9.

improvement in performance, and its ZT value at $300 \mathrm{~K}$ was about 0.025 . Our $\mathrm{Sm}_{2} \mathrm{O}_{3}$-containing sample showed a ZT value of 0.087 at the same temperature, although the silicide should be evaluated at a higher temperatures of $600 \mathrm{~K}$. Ito et al. ${ }^{6}$ ) added $\mathrm{Y}_{2} \mathrm{O}_{3}$ to $\beta$-FeSi $i_{2}$ and this system's figure of merit was $0.1 \times 10^{-3}$, whereas that of our system was $0.49 \times 10^{-3}$ at $600 \mathrm{~K}$. One reason for the increase in the figure of merit is the lower thermal conductivity of the added oxide itself; the thermal conductivities of $\mathrm{Sm}_{2} \mathrm{O}_{3}$ and $\mathrm{Y}_{2} \mathrm{O}_{3}$ are 1.36 and $2.65 \mathrm{~W} \mathrm{~m}^{-1} \mathrm{~K}^{-1}$, respectively.

\section{Conclusion}

We studied ways of improving the thermoelectric properties of $\mathrm{n}-\mathrm{FeSi}_{2}$ by adding an oxide and improved the performance of this material. We regarded to unify the parameters attributing to thermoelectric behaviors, and we found that the reduction potential $(\zeta)$ between the constituent elements of the material is related to the thermoelectric power $(\alpha)$, the electrical resistivity $(\rho)$, and the thermal conductivity $(\kappa)$. We discussed the effectiveness and mechanism of the improvement in the thermoelectric effect achievable by adding an oxide to iron silicide. The addition of $\mathrm{Sm}_{2} \mathrm{O}_{3}$ resulted in the best performance, giving a figure of merit of $0.6-0.7 / \mathrm{K}(\mathrm{ZT}=0.6)$ and a reduction in thermal conductivity by $1 / 3$ compared with standard $n-\mathrm{FeSi}_{2}$; this illustrates the importance of decreasing the value of $\kappa_{\mathrm{ph}}$ and increasing that of $\kappa_{\mathrm{e}}$.

\section{REFERENCES}

1) K. Uemura and I. Nishida: Thermoelectric Semiconductor and Application, (Nikkan Kogyo Shinbun-sha, Tokyo, 1988) p. 178 (in Japanese).

2) I. Nishida: Phys. Rev. B 7 (1973) 2710.

3) S. Sugihara, S. Kawasima, H. Katanahara, H. Suzuki, S. Mochizuki and R. Sekine: Proc. 18th Int. Conf. on Thermoelectrics, 1999, Baltimore, MD, (IEEE, Piscataway, NJ, 1999) p. 577.

4) S. Sugihara, S. Mochizuki, H. Suzuki, T. Bak and J. Nowotny: J. Aust. Ceram. Soc. 37 (2001) 33.

5) S. Sugihara, S. Kawashima, S. Mochizuki, H. Katanahara and H. Suzuki: Proc. 19th Int. Conf. on Thermoelectrics, 2000, Cardiff, UK, (Babrow Press, Cardiff, 2001) p. 398.

6) M. Ito, T. Tada and S. Hara: J. Alloy. Compd. 408-412 (2006) 363.

7) H. Katanahara and S. Sugihara: Proc. 19th Int. Conf. on Thermo- 
electrics, 2000, Cardiff, UK, (Babrow Press, Cardiff, 2001) p. 443.

8) K. Morikawa, H. Chikauchi, H. Mizoguchi and S. Sugihara: Mater. Trans. 48 (2007) 2100-2103.

9) J. Emsley: The Elements, 3rd ed., (Clarendon, London, 1998).

10) M. Hasaka, T. Morimura, T. Watanabe, Y. Etoh and H. Nakashima: Proc. 24th Int. Conf. on Thermoelectrics, 2005 (ICT '05), Clemson, SC, USA, (IEEE, Piscataway, NJ, 2005) p. 419.

11) K. Kurosaki, H. Muta and S. Yamanaka: Proc. Thermoelectric Society of Japan 2004, (Thermoelectric Society of Japan, Fujisawa, 2004) p. 24

12) Y. Aida, H. Muta, K. Kuroaki and S. Yamanaka: Proc. Thermoelectric
Society of Japan 2006, (Thermoelectric Society of Japan, Fujisawa, 2006) p. 14.

13) G. Mahan, B. Sales and J. Sharp: Phys. Today 50 (1997) 42.

14) S. Sugihara, K. Nishiyama, Y. Igarashi and K. Morikawa: Adv. Quantum Chem. 54 (2008) 227.

15) A. F. Ioffe: Semiconductor Thermoelements and Thermoelectric Cooling, (Infosearch, London, 1957).

16) N. W. Ashroft and N. D. Mermin: Solid State Physics, (Saunders College Publishing, New York, NY, 1975) p. 255.

17) I. Ohsugi: Thermoelectric Society of Japan, Newsletter. 3 (1999) 7. 